\title{
The planet companion around $\beta$ Pictoris
}

\author{
Anne-Marie Lagrange ${ }^{1}$, Mickaël Bonnefoy ${ }^{1}$, Gael Chauvin ${ }^{1}$, \\ Daniel Apai ${ }^{2}$, David Ehrenreich ${ }^{1}$, Anthony Boccaletti ${ }^{3}$, \\ Damien Gratadour ${ }^{3}$, Daniel Rouan ${ }^{3}$, David Mouillet ${ }^{1}$, \\ Sylvestre Lacour ${ }^{3}$ and Markus Kasper ${ }^{4}$ \\ ${ }^{1}$ Laboratoire dAstrophysique de Grenoble, France \\ email: lagrange@obs.ujf-grenoble.fr, bonnefoy@obs.ujf-grenoble.fr, \\ chauvin@obs.ujf-grenoble.fr, ehrenreich@obs.ujf-grenoble.fr, \\ mouillet@obs.ujf-grenoble.fr, \\ ${ }^{2}$ Space Telesope Sciente Institute, 3700 San Martin Dr. Baltimore, MD 21218, USA \\ email: apai@stsci.edu \\ ${ }^{3}$ LESIA, Observatoire de Paris, place Jules Janssen, 92195 Meudon, France \\ email: anthony.boccaletti@obspm.fr, \\ daniel.rouan@obspm.fr, damien.gratadour@obspm.fr, sylvestre.lacour@obspm.fr \\ ${ }^{4}$ ESO, Karl Schwarzschild St, 2, 85748 Garching bei Muenchen, Germany \\ email: mkasper@eso.org
}

\begin{abstract}
The $\beta$ Pic disk of dust and gas has been regarded as the prototype of young planetary systems since the 1980s and has revealed over the years an impressive amount of indirect signs pointing toward the presence of at least one giant planet. We present here the recently detected first giant planet around this star. We show how this planet could explain some very peculiar features of the star environment (disk, spectroscopic variability), and how it constrains the scenarios of planetary system formation (timescales, mechanisms).
\end{abstract}

Keywords. planetary systems: formation, techniques: high angular resolution

\section{Planet formation processes}

Understanding planetary systems formation and evolution has become one of the biggest challenges in astronomy, since the imaging of a debris disk around $\beta$ Pictoris, in the eighties and the discovery of the first exoplanet around the solar-like star $51 \mathrm{Pe}-$ gasi during the 90 's. While $\simeq 500$ close planets have been identified with radial velocity (RV) and transit technics, very few have been imaged and definitely confirmed (see Fig. 1 for the closest ones). These few cases already bring new insights in the way planets form. Indeed, while there are now strong pieces of evidence that short period $(\leqslant 5 \mathrm{AU})$ planets detected by RV have formed through core-accretion (CA), the origins of the directly imaged giant planets around stars/brown dwarfs remain debated. If the companions on very wide orbits (a few hundreds of AUs) formed in situ, the acting mechanism was probably stellar-like gaseous collapse, like binaries. This may be true also for 2M1207 b. In such cases, they would be more similar to brown dwarfs rather than planets. Based on various arguments (dynamical timescales, mass of planetesimals available at the current separations), an in-situ formation of HR 8799 bcd and A PsA b would rather involve instabilities (GI) within a circumstellar disk. Planetary-mass bodies might then form in different ways, depending on the initial conditions and in particular the mass of the parent star.

The physics of young giant planets is also still debated. Their brightness, predicted before by so-called "hot-start" models (Baraffe et al. 2003), has been recently questionned 
at young ages (Fortney et al. 2008). The latter predict planets $\simeq 5$ mag. fainter at young ages than the "hot start" model. According to Fortney et al. differences in brightness are present until 50-100 Myr. The difference between both models is due to the modeling of the gas initial properties (temperature), depending on whether the accreting gas energy is radiated away or not. The predictions of planets luminosities is obviously a key issue for planet detection in imaging, especially for the forthcoming planet imagers (GPI, SPHERE) and direct detection and characterization of planets are of crucial importance to calibrate these models.

\section{Discovery of $\beta$ Pictoris b}

In a new analysis of VLT/NACO high dynamics L'-band data of $\beta$ Pictoris taken in Nov. 2003, we detected a faint, point-like signal at $\simeq 8 \mathrm{AU}$ (proj. sep.) from the star, within the North-East side of the dust disk (Fig. 2; Lagrange et al. 2009a). We showed that this candidate companion was probably not a contaminant, but the data alone were not sufficient to clearly rule out this possibility. If bound, its $L^{\prime}=11.2 \pm 0.3$ magnitude would indicate a temperature of $\sim 1500 \mathrm{~K}$ and a mass of $\sim 9 \mathrm{M}_{J u p}$ according to Lyon's group models. Follow-up observations in Jan. and Feb. 2009 (Lagrange et al. 2009b) did not detect the companion, in agreement with either the background hypothesis or the "planet" scenario.

In the fall of 2009, new images revealed a point-source in the SW side of the disk, 0.3 " (i.e; less than $6 \mathrm{AU}$ in proj. sep.) from the star, with a brightness consistent with that of the 2003 source (Fig. 2; Lagrange et al. 2010). These observations also definitely showed that the 2003 source was not a background source and is indeed orbiting $\beta$ Pictoris. Its 2009 location furthermore implies a semi-major axis of about 8-15 U. It shows that the planet was observed after quadrature in 2003. While more precise orbital parameters are still to be determined by a careful orbital follow-up, $\beta$ Pictoris b is now the closest planet ever imaged around a star. More recently, $\beta$ Pictoris b was also detected at 4 microns with the recently available APP device on NACO (Quanz et al. 2010) as well as at Ks (Bonnefoy et al. 2011). In both cases, the constraints derived on the planet effective temperature are in agreement with those brought by the L' data.

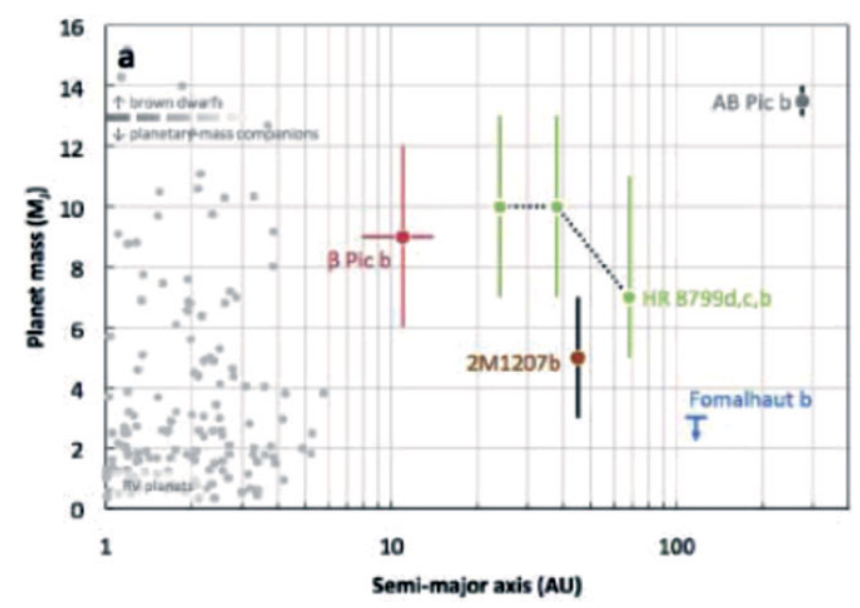

Figure 1. Planetary mass objects imaged close ( $\leqslant 120$ AUs) to stars (as of October 2010). 


\section{Constraints brought by $\beta$ Pictoris b}

Constraints on planet formation It is well accepted that giant planet formation should occur rapidly (less than a few Myr, as inferred from studies of dispersal timescales of young gaseous disks). Given the system young age (12 $2_{-4}^{+8} \mathrm{Myr}$; Zuckerman et al. 2001), $\beta$ Pictoris b now proves directly for the first time that giant planets can form in Myr time-scales in disks. Based on current models predictions according to its parent star's properties (age, star mass) developped by Kennedy and Kenyon (2008), and given its properties, $\beta$ Pictoris $\mathrm{b}$ is probably the first planet imaged that could have formed via core accretion, like, our Solar System giant planets. It offers then the opportunity to constrain CA-related models in the future. We already note that the "cold start" evolutionary model proposed by Fortney et al.(2008) fails to reproduce its luminosity.

$\beta$ Pictoris disk While about 20 debris disks - disks containing dust produced by collisions among larger rocky bodies - have been optically resolved today, $\beta$ Pictoris remains the best-studied young system, with several indirect signs of the presence of planets on circular or low eccentric orbit (see a review in Lagrange et al. (2000), Freistetter et al. (2007)). Among the numerous peculiarities of the disk, is the so-called inner $(\simeq 60-80$ AU) 5-6 degrees warp (Mouillet et al. 1997; Heap et al. 2000; Golimowski et al. 2004). More than 10 years ago, we interpreted and modeled this warp as the result of the gravitational perturbation of a massive body located on an inclined orbit, on a disk of planetesimals (Mouillet et al. 1997; Augereau et al. 2001). Such a configuration also explained the observed butterfly asymmetry (Kalas and Jewitt 1995). We derived in 1997 an analytical relation between the warp extension and the perturbing body properties. When using updated values of the star age and warp characteristics, it appears that the characteristics of $\beta$ Pictoris $\mathrm{b}$ do verify this relation. However, within the present error bars, we cannot determine whether or not it is located in the inner warped disk or not. We note that if further observations show that the companion lies indeed in the warped disk, between 8 and $14 \mathrm{AU}$, then we will have a direct (independant from evolutionary models) constraint on its mass (to be less than 42 MJup).

Very interestingly, $\beta$ Pictoris exhibits a very peculiar spectroscopic variability (see e.g. Vidal-Madjar et al. 1998; Lagrange et al. 2000) that has been since many years

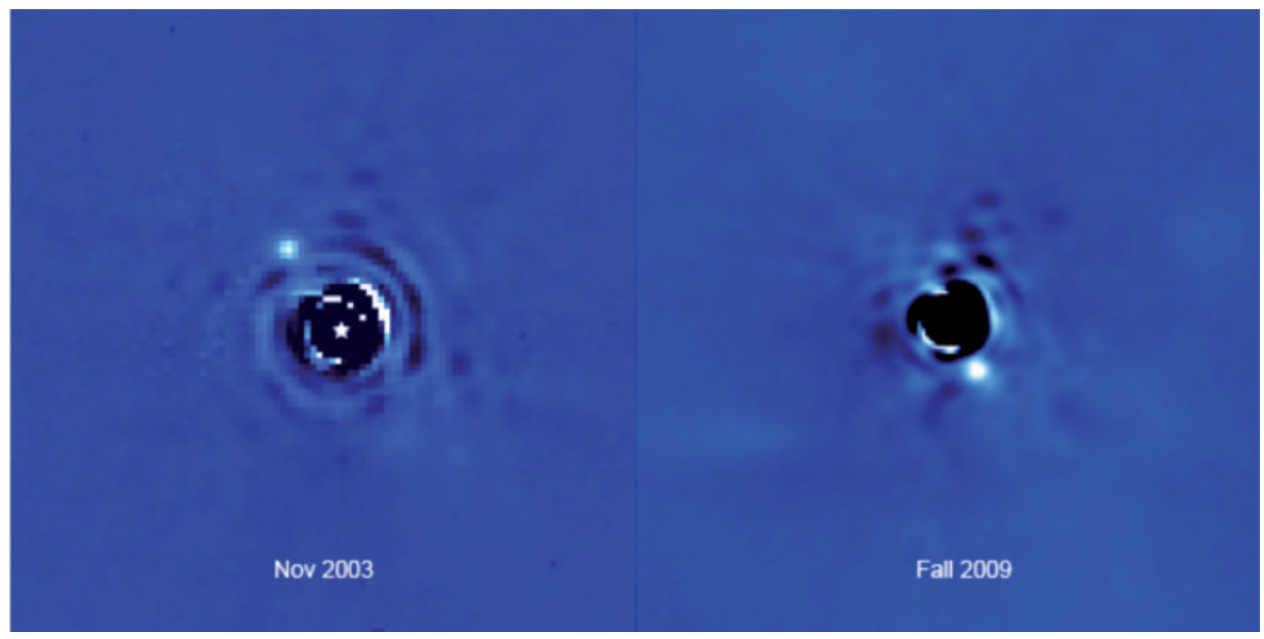

Figure 2. L' VLT/NACO images of $\beta$ Pictoris b in 2003 and 2009. In both images, North is up and East is to the left. In Nov 2003, $\beta$ Pictoris b lies 0.4" NE from the star, and in Fall 2009, 0.3 " SW from the star. 
attributed to the evaporation of star grazing comets (FEBs; Beust \& Morbidelli 2005 and ref therein). $\beta$ Pictoris b could be at the origin of the observed cometary infall.

Finally, Lecavelier des Etangs \& Vidal-Madjar (2009) showed that the candidate companion observed in 2003 could be responsible for the peculiar photometric variation observed in 1981, provided its semi-major axis (circular case or assuming a low eccentricity) is in the range 7.6-8.7 AU, corresponding to periods in the range 16-20 years. The 2009 imaging data are still compatible with such a scenario. If confirmed, we could have access in a few years, to the transmission spectrum of a transiting giant planet located beyond the ice zone.

\section{References}

Augereau, J. C., Nelson, R., Lagrange, A. M., et al. 2001, A\&̊A , 370, 447

Baraffe, I., Chabrier, G., Barman, T. S., et al. 2003, A\&SA, 402, 701

Beust, H. \& Morbidelli, A. Icarus, 143, 170

Beuzit, J.-L., Boccaletti, A., Feldt, M., et al. 2010, ASPC, 430, 231

Bonnefoy, M., Lagrange, A. M., Chauvin, G. et al. 2011, A\&A , 528, L15

Fortney, J. J., Marley, M. S., Saumon, D., \& Lodders, K. 2008, ApJ , 683, 1104

Freistetter, F, Krivov, A. V. \& Loehene, T. 2007, A\&SA , 466, 389

Golimovski, D. A., Henry, T. J., Krist, J. E., et al. 2004, AJ , 128, 1733

Heap, S., Lindler, D. J., Lanz, T. M., et al. 2000, ApJ , 539, 435

Kalas, P. \& Jewitt, D. 1995, AJ , 110, 794

Kennedy, G. M. \& Kenyon, S. J. 2008, ApJ 673, 502

Lagrange, A.-M., Backman, D., \& Artymowicz, P. 2000, in Protostars 83 Planets IV, Univ. of Arizona Press, 639

Lagrange, A.-M., Gratadour, D., Chauvin, G., et al. 2009a, A\&A , 493, L21

Lagrange, A.-M., Kasper, M., \& Boccaletti, A., et al. 2009b, A\&A , 506, L927

Lagrange, A.-M., Bonnefoy, M., Chauvin, G., et al. 2010, Science, 329, 57

Lecavelier des Etangs, A. \& Vidal-Madjar, A. 2009, A\& A , 497, 557

Quanz, S., Meyer, M. R., Kenworthy, M. A., et al. ApJ , 722, L49

Mouillet, D., Larwood, J. D., Papaloizou, J. C. B., \& Lagrange A.-M. 1997, MNRAS , 292, 896

Vidal-Madjar, A., Lecavelier des Etangs, A., \& Ferlet, R. 1998, Planetary \& Space Science, 46, 629

Zuckerman, B., Song, I., Bessel, M. S., \& Webb, R. A. 2001, ApJ , 562, L87 